\title{
Crenosoma vulpis in dog: first case report in Italy and use of the FLOTAC technique for copromicroscopic diagnosis
}

\author{
L. Rinaldi • G. Calabria • S. Carbone • A. Carrella • \\ G. Cringoli
}

Published online: 10 January 2008

(C) Springer-Verlag 2008

Erratum to: Parasitol Res (2007) 101:1681-1684

DOI 10.007/s00436-006-0713-6

The original version of this article unfortunately contained a mistake. The presentation of Table 1 was incorrect. The corrected table is given below.

Table 1 LPG values (mean, standard error, and percentiles) of Crenosoma vulpis detected by the five copromicroscopic techniques

\begin{tabular}{|c|c|c|c|c|c|c|}
\hline \multirow[t]{3}{*}{ Copromicroscopic techniques } & \multirow[t]{3}{*}{ Multiplication factor } & \multicolumn{5}{|c|}{ LPG (no.=10 replicates) } \\
\hline & & \multirow[t]{2}{*}{ Mean* } & \multirow[t]{2}{*}{ SE } & \multicolumn{3}{|c|}{ Percentiles } \\
\hline & & & & $25^{\text {th }}$ & $50^{\text {th }}$ & $75^{\text {th }}$ \\
\hline FLOTAC & 2 & $91.3^{\mathrm{a}}$ & 5.1 & 82.0 & 88.0 & 104.0 \\
\hline McMaster & 10 & $36.7^{\mathrm{b}}$ & 6.1 & 27.5 & 30.0 & 52.5 \\
\hline Baermann & 0.1 & $0.7^{\mathrm{c}}$ & 0.05 & 0.6 & 0.7 & 0.8 \\
\hline Flotation & 1.6 & $11.1^{\mathrm{c}}$ & 0.5 & 10.0 & 10.8 & 12.8 \\
\hline Wisconsin & 1.6 & $36.7^{\mathrm{b}}$ & 6.7 & 31.6 & 36.7 & 43.3 \\
\hline
\end{tabular}

*Significant differences for different letters $(P<0.05)$.

The online version of the original article can be found at http://dx.doi. org/10.1007/s00436-007-0713-6.

L. Rinaldi $\cdot$ S. Carbone $\cdot$ G. Cringoli

Dipartimento di Patologia e Sanità Animale,

Università degli Studi di Napoli "Federico II"-CREMOPAR

Regione Campania,

Naples, Italy

\section{G. Cringoli $(\bowtie)$}

Dipartimento di Patologia e Sanità Animale,

Facoltà di Medicina Veterinaria,

Università degli Studi di Napoli "Federico II",

Via della Veterinaria,

1.80137 Naples, Italy

e-mail: cringoli@unina.it
Present address:

G. Calabria $\cdot$ A. Carrella

Marigliano,

Naples, Italy 